\title{
Radiative Transfer of CO through Clumpy Molecular Clouds with External UV Heating
}

\author{
Jan A. Tauber ${ }^{1}$ and Paul F. Goldsmith ${ }^{2}$ \\ ${ }^{1}$ Radio Astronomy Laboratory, Univ. of California, Berkeley, CA \\ ${ }^{2}$ Five College Radio Astronomy Observatory, Univ. of Massachusetts, \\ Amherst, MA.
}

We have developed a model which simulates the radiative transfer of molecular line emission through clumpy molecular clouds. The dynamical structure of the model cloud is based on the work of Kwan and Sanders (1986). The model incorporates the existence of an intense source of UV photons at the surface of the cloud. The UV source heats the clumps and creates kinetic temperature and $\mathrm{CO}$ abundance gradients within them. The amount of heating depends on the intensity of the UV field, which decreases from the surface to the core of the cloud due to attenuation by dust. We treat in detail the photochemistry and selfshielding properties of $\mathrm{CO}$ as a function of UV intensity and gas density in order to obtain the $\mathrm{CO}$ line intensities emerging from each clump. The line intensity emerging from the cloud is obtained by integrating the emission from all clumps along the line of sight, weighted by an area covering factor, and attenuated by the opacity of intervening clumps. The effects of the heating are significantly noticeable on the line intensities of $\mathrm{CO}$ transitions arising from levels with $\mathrm{J}$ between 3 and $\sim 7$. We apply our model to the case of the Orion A molecular cloud, and in particular to observations of the $\mathrm{J}=3 \rightarrow 2{ }^{12} \mathrm{CO}$ and ${ }^{13} \mathrm{CO}$ lines. The model is in general agreement with the observed enhanced intensity of the ${ }^{12} \mathrm{CO} \quad \mathrm{J}=3 \rightarrow 2$ transition relative to the $\mathrm{J}=1 \rightarrow 0$ transition throughout the central $\sim 10^{\prime}$ region of Orion. It also produces centrally peaked spectral lines whose intensity is maximum in a shell-like distribution centered on the Trapezium HII region, as is observed.

\section{References}

Kwan, J., and Sanders, D.B. 1986, Ap.J., 309, 783 